THEORY OF THE GIMMICK 



\title{
THEORY OF THE GIMMICK
}

Aesthetic Judgment and Capitalist Form

\author{
Sianne Ngai
}

THE BELKNAP PRESS OF

HARVARD UNIVERSITY PRESS

Cambridge, Massachusetts

London, England

2020 
Copyright ( 2020 by the President and Fellows of Harvard College All rights reserved Printed in the United States of America

First printing

Cover photograph: Flame, 1947 @ Stan Douglas

Cover design: Tim Jones

$$
\begin{gathered}
9780674245310 \text { (EPUB) } \\
9780674245341 \text { (MOBI) } \\
9780674245365 \text { (PDF) }
\end{gathered}
$$

The Library of Congress has cataloged the printed edition as follows:

Names: Ngai, Sianne, author.

Title: Theory of the gimmick : aesthetic judgment and capitalist form / Sianne Ngai.

Description: Cambridge, Massachusetts : The Belknap Press of Harvard University Press, 2020. I Includes bibliographical references and index.

Identifiers: LCCN 2019038757 | ISBN 9780674984547 (cloth)

Subjects: LCSH: Aesthetics, Modern-20th century. I

Aesthetics, Modern-21st century. | Aesthetics, Modern—Philosophy. I

Hoaxes. I Influence (Literary, artistic, etc.) I Capitalism.

Classification: LCC BH201 .N49 2020 I DDC 111/.85-dc23

LC record available at https://lccn.loc.gov/2019038757 
For Hans Thomalla 
